\title{
Search for new physics beyond the Standard Model in final states with jets and leptons+jets at CMS
}

\author{
Francesco Santanastasio ${ }^{1, a}$ on behalf of the CMS collaboration \\ ${ }^{1}$ Sapienza, University of Rome and INFN Rome
}

\begin{abstract}
Searches for new physics beyond the Standard Model performed by the CMS experiment in final states with jets and leptons+jets are presented. The document focuses on a selection of recent results from searches for heavy dijet resonances, leptoquarks, and heavy majorana neutrinos using proton-proton collision data at both $\sqrt{s}=8$ and $13 \mathrm{TeV}$. The novel CMS datascouting technique, used to probe the low-mass region in hadronic final states, is introduced and results from a Run1 analysis are shown. Two searches in the electrons+jets final states showing an excess of events in Run1 data are also discussed.
\end{abstract}

\section{Introduction}

Searches for new physics beyond the Standard Model performed by the CMS experiment [1] at the CERN LHC are presented in these conference proceedings. A selection of searches in final states with jets and leptons+jets is shown ${ }^{1}$. Results are obtained using proton-proton collision data collected in Run1 (2012) at $\sqrt{s}=8 \mathrm{TeV}$ and Run2 (2015) at $\sqrt{s}=13 \mathrm{TeV}$. Section 2 presents results of the search for dijet resonances at high-mass using 2015 Run2 data and results of the low-mass analysis with the Run1 data scouting technique. Section 3 reports the results from searches for leptoquarks at $\sqrt{s}=13 \mathrm{TeV}$. In Section 4 a few interesting excesses of events in data observed in the Run1 searches for leptoquarks and heavy majorana neutrinos are discussed. Finally the future prospects for these searches are presented in Section 5.

\section{Dijet resonances}

The search for new resonances decaying to pairs of jets is among the most important ones at LHC because any hypothetical new particle that might be produced originates from the colliding protons and therefore it must couple to quarks and/or gluons.

The analysis strategy consists in reconstructing the invariant mass of the dijet system $\left(\mathrm{m}_{\mathrm{jj}}\right)$ and searching for a resonant peak in its spectrum. Wide-jets (corresponding to a jet cone size of $\Delta R=\sqrt{\Delta \eta^{2}+\Delta \phi^{2}}=1.1$ ) are used to reconstruct the energy of the initial partons from the resonance decay. This algorithm improves the energy resolution by collecting final state radiation from

\footnotetext{
ae-mail: francesco.santanastasio@cern.ch

${ }^{1}$ The complete list of publications and preliminary results on these subject are available in the CMS public web pages [2].
} 
the outgoing partons. Events used for the search are required to have $\Delta \eta_{j j}=\left|\eta_{j 1}-\eta_{j 2}\right|<1.3$ reducing significantly the QCD multijet background in t-channel with respect to the s-channel process of resonance production. The search is performed in two different dijet mass regions depending on the trigger strategy used: high-mass $\left(\mathrm{m}_{\mathrm{jj}}>1 \mathrm{TeV}\right)$ and low-mass $\left(500 \mathrm{GeV}<\mathrm{m}_{\mathrm{jj}}<1 \mathrm{TeV}\right)$.

\subsection{High-mass dijet search}

The high-mass analysis uses events collected by standard hadronic triggers employed in many CMS analyses. The trigger requires that events have $H_{T}>800 \mathrm{GeV}$, where $H_{T}$ is the scalar sum of all jets with $p_{T}>40 \mathrm{GeV}$ reconstructed at high level trigger (HLT). Offline events are required to pass a cut on the dijet mass at approximately $\mathrm{m}_{\mathrm{jj}}>1 \mathrm{TeV}$ for which the trigger is fully efficient on events in data passing the final dijet selection.

Figure 1 (left) shows a display of the highest dijet-mass event passing the full selection, recorded by CMS during 2015 data taking. The reconstructed invariant mass of the dijet system is about $6 \mathrm{TeV}$. Figure 1 (right) shows the dijet mass spectrum in $2.4 \mathrm{fb}^{-1}$ of data collected in 2015 at $\sqrt{s}=13 \mathrm{TeV}$ [3]. The background is estimated by fitting the data with a smoothly falling function. The parametrization used is

$$
\frac{d \sigma}{d \mathrm{~m}_{\mathrm{jj}}}=\frac{P_{0}(1-x)^{P_{1}}}{x^{P_{2}+P_{3} \ln (x)}}
$$

where $x=\mathrm{m}_{\mathrm{jj}} / \sqrt{s}$. This function models well the QCD multijet simulation and the data and it has been successfully employed in previous searches at hadron colliders at different $\sqrt{s}$ values. No sign of new resonances is observed in the dijet mass spectrum, and 95\% confidence level (CL) upper limits are set on the resonance production cross section $(\sigma)$ time branching ratio to dijet $(B R)$ times acceptance $(A)$ as a function of the hypothesized resonance mass. The limits are reported in Figure 2 (left) for different final states (quark-quark, quark-gluon, gluon-gluon depending on the resonance decay products). The upper limits on $\sigma \times B R \times A$ are compared to theoretical predictions for several new physics models including strongly coupled resonances (such as string resonances, excited quarks, axigluons, etc.) and weakly coupled resonances (such as Z' and ' '' bosons and Randall-Sundrum Gravitons), covering a wide range of cross sections to which this analysis is sensitive. Lower limits are set on the mass of these resonances as summarized in Figure 2 (right). The limits of this analysis significantly exceed the ones from the previous Run1 search thanks to the increased center-of-mass energy in Run2.

\subsection{Low-mass dijet search with data scouting}

It is important to extend the dijet search in the mass region below $1 \mathrm{TeV}$ in order to probe hypothetical hadronic resonances with small couplings to quarks and gluons that similar searches performed at previous colliders could not find yet. The main experimental difficulties at LHC originate from the large cross section of multijet events at low dijet mass and the finite computing resources for processing and storing these data. To solve this issue, a new technique known as data scouting was first proposed by CMS in 2011 [4]. This technique, by significantly reducing the event size compared to the standard CMS data stream (about a factor 100), enabled CMS to relax the trigger thresholds and record fully hadronic events at a rate of few $\mathrm{KHz}$ to extend the search in the sub-TeV mass region. With this approach, the analysis is performed using jets reconstructed online in the CMS trigger computing farm.

This novel research strategy was fully integrated in the CMS physics program in 2012 [5] allowing to collect data corresponding to almost $19 \mathrm{fb}^{-1}$ of integrated luminosity at $\sqrt{s}=8 \mathrm{TeV}$ in the sub-TeV 

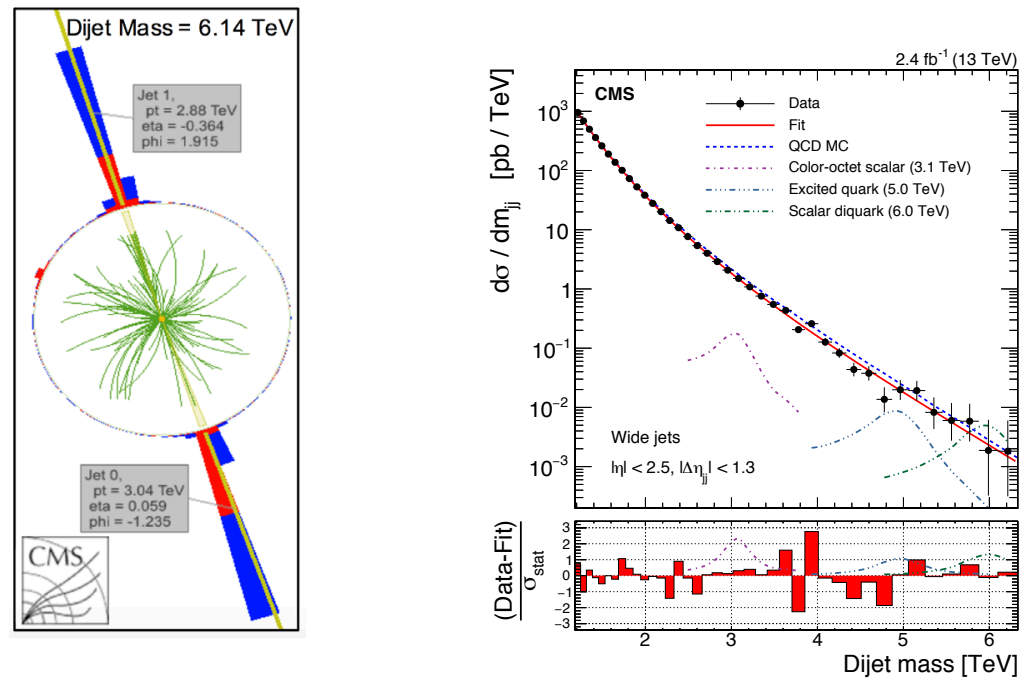

Figure 1. Left: Display of the event with the highest dijet mass $(6.14 \mathrm{TeV})$ after the full selection. The transverse view with respect to the beam axis is shown. The kinematic quantities of the two wide jets are reported. Right: Dijet mass spectrum (points) compared to a fitted parameterization (solid curve) and to the prediction of the PYTHIA8 QCD MC event generator including simulation of the detector (dashed curve). The lower panel shows the difference between the data and the fitted parametrization, divided by the statistical uncertainties. The predicted distributions of narrow resonance signals for three models, with resonance mass values corresponding to the respective $95 \%$ confidence level exclusion limit, are shown in both panels (dash-dotted curves).

dijet mass region. The new trigger allows to go down to $400 \mathrm{GeV}$ in dijet mass. The offline analysis strategy used for the high-mass analysis is also employed in this low-mass search. Figure 3 (left) shows the dijet mass spectrum in scouting data in the region between 400 and $1900 \mathrm{GeV}$. No sign of new resonances in observed and upper limits on $\sigma \times B R \times A$ are set as a function of the resonance mass. The analysis results has been published on the PRL journal in 2016 [6].

It is interesting to compare the limits of this search with those obtained by previous experiments at hadronic colliders with different center-of-mass energies. This can be done assuming a common theoretical model of a new resonance $Z$ ' that decays to a pair of quarks as described in Ref. [7]. This simplified model is characterized by only two free parameters: the resonance mass $M_{Z^{\prime}}$ and the coupling $g_{B}$ of the resonance to the quarks. The upper limits on signal cross section can be translated into limits in the coupling vs mass plane as shown in Figure 3 (right). The CMS dijet analysis with data scouting extends the search below $1 \mathrm{TeV}$ and provides, at the time of the publication, the most stringent limits to date in the region between 500 and $800 \mathrm{GeV}$. In the region below $500 \mathrm{GeV}$ the best limits still come from CDF and UA2 experiments. ATLAS and CMS have initiated recently a physics program targeting the mass region below $500 \mathrm{GeV}$ using various techniques that exploit the emission of initial state radiation (quarks/gluons or photons) to trigger these events. 

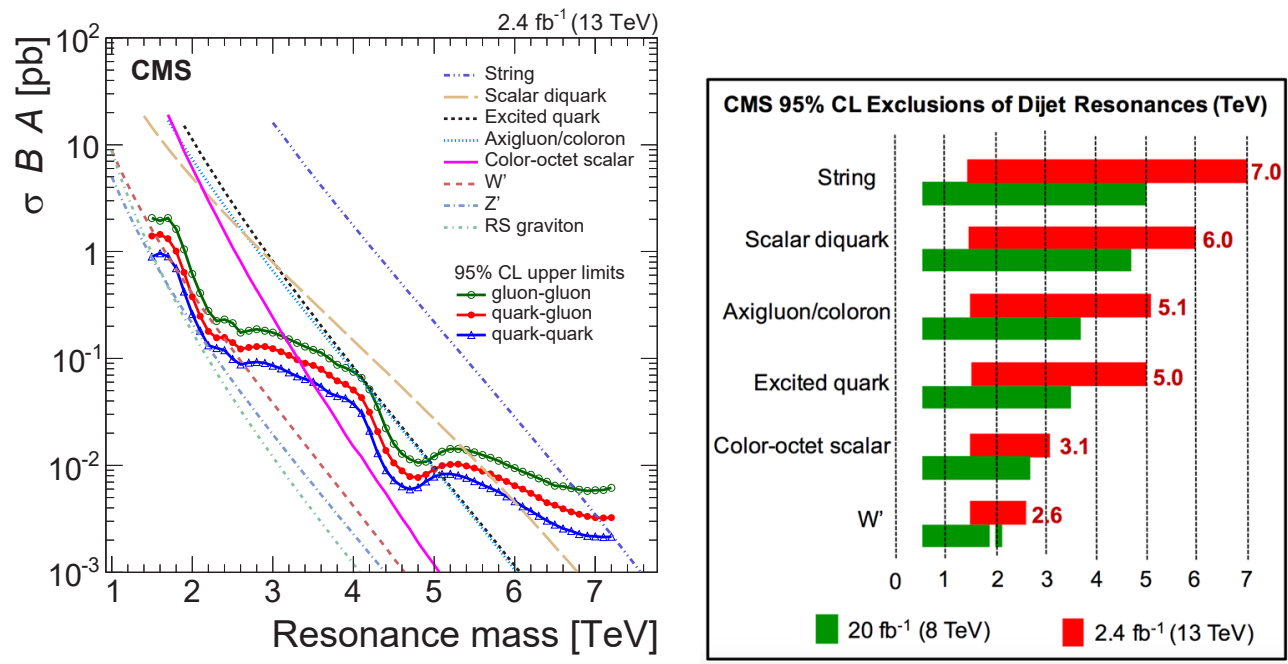

Figure 2. Left: The observed 95\% CL upper limits on the product of the cross section, branching fraction, and acceptance for quark-quark, quark-gluon, gluon-gluon type dijet resonances. The limits are compared to the predicted cross sections of several resonance models. Right: Observed 95\%CL lower limits on resonance mass for the listed models obtained by the CMS experiment in Run 1 (green) and Run 2 (red) analyses.
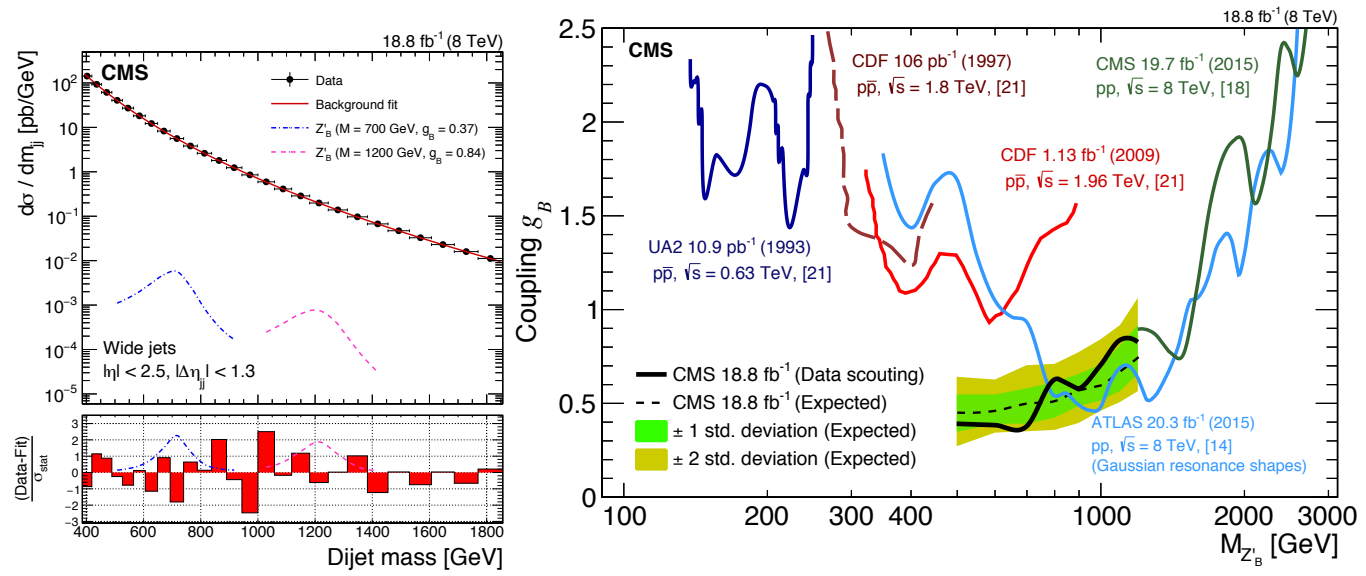

Figure 3. Left: Dijet mass spectrum using data scouting (points) compared to a fitted parameterization (solid curve). The bottom panel compares the data and the fit result, normalized by the statistical uncertainty in the data, for each bin. The predicted distributions of narrow resonance signals for a hypothetical leptophobic resonance Z' with two different mass and coupling values are shown in both panels (dash-dotted curves). This dijet mass distribution complements that observed in the high-mass analysis. Right: Observed 95\% CL upper limits on the coupling $g_{B}$ of the Z' resonance as a function of its mass. The results are compared to those obtained with similar searches at different collider energies. 


\section{Leptoquarks}

The Standard Model contains hints that the lepton and quark sectors are related by a fundamental symmetry. Many beyond the Standard Model theories (such as grand unified theories, composite models, technicolor, SUSY RPV, and others) include such a symmetry, and predict the existence of new massive bosons called leptoquarks (LQ). Leptoquarks can have spin 0 or 1 , are coloured particles, have fractional electric charge, and carry both baryon and lepton number. In order to avoid proton decay, baryon and lepton numbers must be conserved separately. In addition constrains from rare decays involving flavour-changing-neutral-currents suggests that LQs should only couple with leptons and quarks within the same generation.

In the simplest case, the model parameters are three: the mass of the LQ $\left(M_{L Q}\right)$, the branching ratio of the LQ decay into charged lepton and quark $(\beta)$, and the LQ-lepton-quark coupling $(\lambda)$. The branching ratio of the LQ decay into neutrino and quark is $1-\beta$. At LHC, LQs can be produced singly or in pairs. The pair production, which happens mainly via gluon-gluon fusion, is particularly interesting because it does not depend on the unknown coupling $\lambda$. The pair production cross section depends on the strong coupling and it is calculated at next-to-leading order.

Depending on the value of the parameter $\beta$ different decay channels are possible from the pair production of LQs. They differ for the branching ratio $(B R)$ and the observed final state, thus producing a wide range of experimental signatures:

- $\ell \ell j j$ channel, $B R=\beta^{2}$, two charged leptons plus two jets;

- $\ell v j j$ channel, $B R=2 \beta(1-\beta)$, one charged leptons, missing transverse energy (MET) plus two jets;

- $v v j j$ channel, $B R=(1-\beta)^{2}$, two jets plus MET.

Feynman diagrams for the different final states are shown in Figure 4.

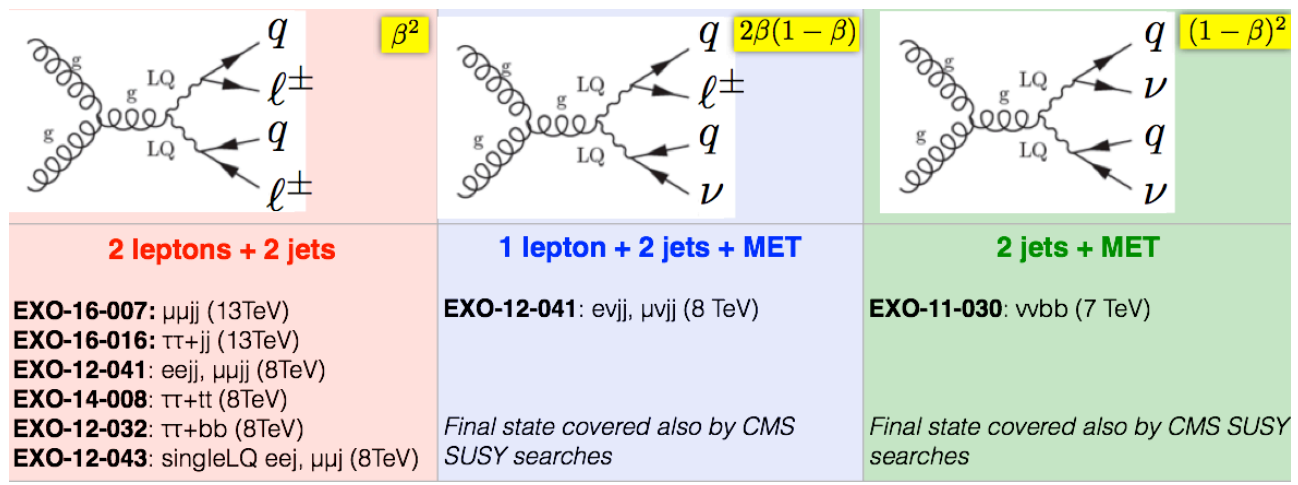

Figure 4. Feynman diagrams for different final states in LQ pair production processes. The labels EXO-XXYYY indicates the identifier of the CMS publications or preliminary results describing the most recent searches in the corresponding channel [2].

The most recent searches for LQ at CMS have been performed using about $2 \mathrm{fb}^{-1}$ of $\sqrt{s}=13 \mathrm{TeV}$ data collected in 2015.

A search for second generation LQs is performed in final states with two muons and two jets [8]. The event selection, optimized for each LQ mass hypothesis, is based on three main kinematic observables: the di-muon invariant mass, the $S_{T}=p_{T}\left(\mu_{1}\right)+p_{T}\left(\mu_{2}\right)+p_{T}\left(j e t_{1}\right)+p_{T}\left(\right.$ jet $\left._{2}\right)$ variable, and the lepton-jet mass $M_{\mu j}^{\min }$ defined as the smaller of the two reconstructed LQ masses which minimizes 
the LQ-LQ mass difference. In presence of a signal, the $M_{\mu j}^{m i n}$ distribution would show a narrow peak in correspondence of the LQ mass, as shown in Figure 5 (left).

A search for third generation LQs is performed in final states with two taus (with reconstructed hadronic decays) and two jets [9]. The main physics observable to discriminate between signal and background is the variable $S_{T}=p_{T}\left(\tau_{1}\right)+p_{T}\left(\tau_{2}\right)+p_{T}\left(j e t_{1}\right)+p_{T}\left(\right.$ jet $\left._{2}\right)$ shown in Figure 5 (right).

No excess of events is observed in data in these searches and lower limits are set on the mass of second and third generation LQs at $1150 \mathrm{GeV}$ and $740 \mathrm{GeV}$, respectively, assuming $\beta=1$.
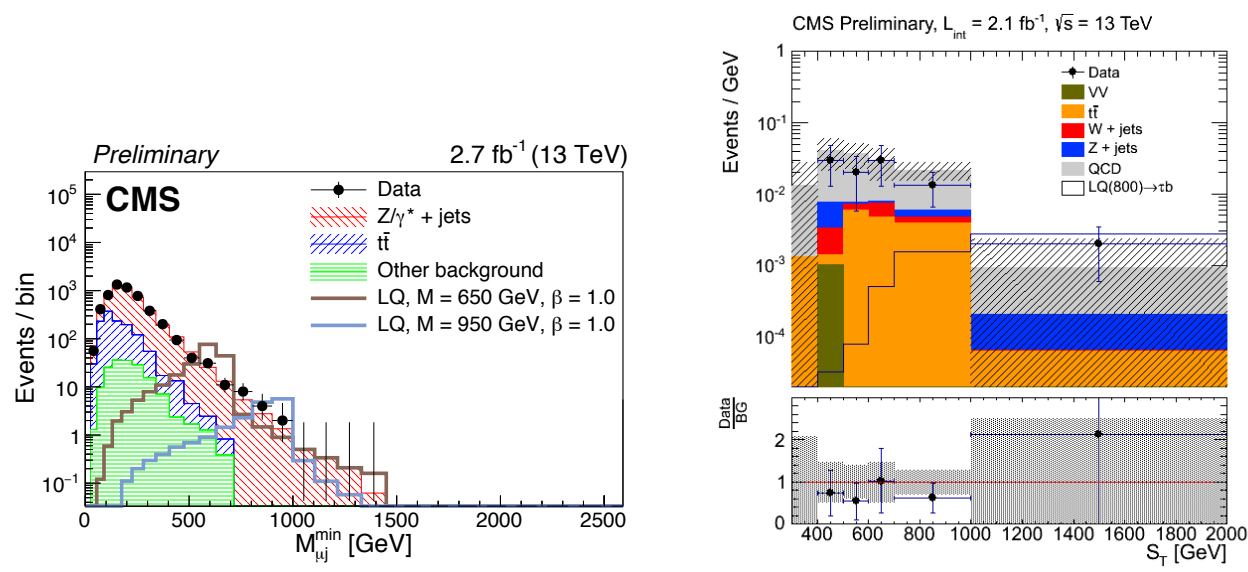

Figure 5. The $M_{\mu j}^{\text {min }}$ distribution in events with two muons and two jets (left). The $S_{T}$ distribution in events with two taus decaying hadronically and two jets (right). Distributions for the data, the background predictions, and the expected LQ signals are shown.

\section{Excess of events in Run1 data}

Two searches for new physics performed in Run1 data at $\sqrt{s}=8 \mathrm{TeV}$ in final states with electrons and jets reported an excess of events in data compared to the background prediction.

\subsection{First generation leptoquarks}

A search for first generation leptoquarks in the $e v j j$ channel have been performed with about $20 \mathrm{fb}^{-1}$ of data [10]. The analysis is designed as a counting experiment in a signal region defined by an event selection optimized for each LQ mass hypothesis. The reconstructed observables used in the selection to separate signal from background are the electron-neutrino transverse mass, the variable $S_{T}=p_{T}\left(e_{1}\right)+M E T+p_{T}\left(\right.$ jet $\left._{1}\right)+p_{T}\left(\right.$ jet $\left._{2}\right)$, and the electron-jet mass $M_{e j}$. Figure 6 (left) shows the electron-jet mass distribution after a selection optimized for the search of a LQ with $650 \mathrm{GeV}$ mass. A broad excess of data events is visible above $600 \mathrm{GeV}$ compared to the background expectation. This reflects also in the upper limits on signal cross section times branching ratio shows in Figure 6 (right) in the same LQ mass region. The local significance of the excess is 2.6 standard deviations. No excess is observed in the $\mu v j j$ channel. 

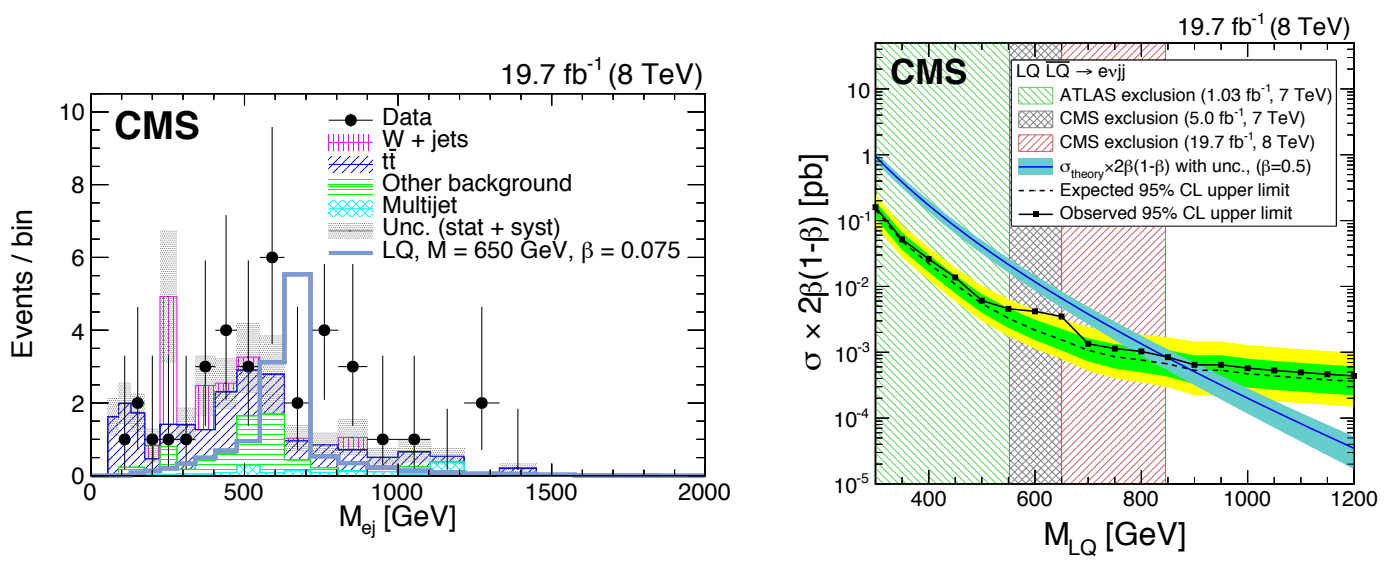

Figure 6. Left: The $M_{e j}$ distribution in the $e v j j$ channel after the selection criteria optimized for a LQ mass of $650 \mathrm{GeV}$ have been applied. Right: the expected and observed upper limits at 95\% CL on the LQ pair production cross section times branching ratio as a function of the first generation LQ mass obtained in the $e v j j$ channel.

\section{2 $W_{R}$ and heavy Majorana neutrinos}

The second analysis is a search for $W_{R}$ and heavy Majorana neutrinos [11]. These new heavy particles are predicted in left-right symmetric extensions of the Standard Model. The model introduces an additional right-handed $\mathrm{SU}_{R}(2)$ symmetry group to the $\mathrm{SM}$, which includes heavy charged $\left(W_{R}^{ \pm}\right)$and neutral $\left(Z_{R}\right)$ gauge bosons in addition to heavy right-handed Majorana neutrinos $\left(N_{e}, N_{\mu}, N_{\tau}\right)$. The latter ones provide a possible explanation for the mass of SM neutrinos through the so called see-saw mechanism. Figure 7 (left) shows a diagram of the production and decay of a massive $W_{R}$ gauge boson decaying to an heavy Majorana neutrino. The final state contains two charged, same-flavour leptons and two jets. In presence of a signal the invariant mass of the four-body system $M_{\ell \ell j j}$ peaks at the mass of the $W_{R}$ boson. Figure 7 (right) shows the $M_{e e j j}$ distribution in data events with two electrons and two jets. An excess is visible at around $2 \mathrm{TeV}$ corresponding to a local significance of 2.8 standard deviations. No excess is observed in the two muons plus two jets channel.

\section{Future prospects}

Searches in jets and leptons+jets final states at CMS are ongoing using the recent 2016 data. A datasample of about $40 \mathrm{fb}^{-1}$ should be recorded at $\sqrt{s}=13 \mathrm{TeV}$ for both ATLAS and CMS experiments. The sensitivity to new physics of the searches presented in this document will improve significantly compared to the current results thanks to a dataset 20 times larger than 2015.

At the date of this conference, the two searches discussed in Section 4 have not being repeated yet at $\sqrt{s}=13 \mathrm{TeV}$ neither by ATLAS or CMS experiments. Although the observed excesses are still compatible with statistical fluctuations of the background, it is very interesting to see if the new analyses will confirm the results observed in the $8 \mathrm{TeV}$ data. New results from the CMS collaboration are expected in 2017. 

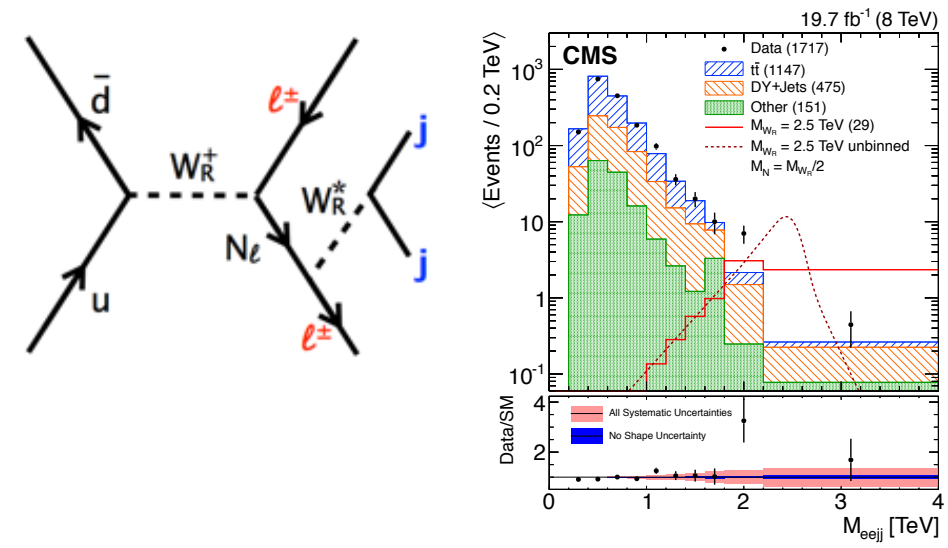

Figure 7. Left: Diagram of production of $W_{R}$ and decay to heavy Majorana Neutrino. Right: $M_{e e j j}$ distribution in events with two electrons and two jets after full selection.

\section{References}

[1] CMS Collaboration, "The CMS experiment at the CERN LHC," JINST 3, S08004 (2008).

[2] https://cms-results.web.cern.ch/cms-results/public-results/publications/EXO/index.html

[3] CMS Collaboration, "Search for narrow resonances decaying to dijets in proton-proton collisions at $\sqrt{(} s)=13$ TeV," Phys. Rev. Lett. 116, no. 7, 071801 (2016), arXiv:1512.01224.

[4] CMS Collaboration, "Search for Narrow Resonances using the Dijet Mass Spectrum in pp Collisions at sqrt s of $7 \mathrm{TeV}$," CMS-PAS-EXO-11-094.

[5] CMS Collaboration, "Data Parking and Data Scouting at the CMS Experiment," CMS-DP-2012022.

[6] CMS Collaboration, "Search for narrow resonances in dijet final states at $\sqrt{(} s)=8 \mathrm{TeV}$ with the novel CMS technique of data scouting," Phys. Rev. Lett. 117, no. 3, 031802 (2016), arXiv:1604.08907.

[7] B. A. Dobrescu and F. Yu, "Coupling-mass mapping of dijet peak searches," Phys. Rev. D 88, no. 3, 035021 (2013), Erratum: [Phys. Rev. D 90, no. 7, 079901 (2014)], arXiv:1306.2629.

[8] CMS Collaboration, "Search for pair-production of second-generation scalar leptoquarks in pp collisions at $\sqrt{s}=13 \mathrm{TeV}$ with the CMS detector," CMS-PAS-EXO-16-007.

[9] CMS Collaboration, "Search for heavy neutrinos and third-generation leptoquarks in final states with two hadronically decaying $\tau$ leptons and two jets in proton-proton collisions at $\sqrt{s}=13 \mathrm{TeV}$," CMS-PAS-EXO-16-016.

[10] CMS Collaboration, "Search for pair production of first and second generation leptoquarks in proton-proton collisions at $\operatorname{sqrt}(\mathrm{s})=8 \mathrm{TeV}$," Phys. Rev. D 93, no. 3, 032004 (2016) arXiv: 1509.03744 .

[11] CMS Collaboration, "Search for heavy neutrinos and W bosons with right-handed couplings in proton-proton collisions at $\sqrt{s}=8 \mathrm{TeV}$," Eur. Phys. J. C 74, no. 11, 3149 (2014) arXiv:1407.3683. 